\title{
12 Wader Counts
}

The phenology of wader migration at stopover sites is the subject of many studies, and forms the basis of other more detailed analysis. In passerine ringing stations, data from catches are frequently used to illustrate migration dynamics or monitor population numbers. In the case of waders, the size of discrepancies in migration patterns obtained according to counts and trapping in walk-in traps differed between years, and it was related to neither the aggregate number of migrants counted nor the total caught. However, it would seem likely that in the case of a very rare species, the dates on which birds are trapped would show little correlation with migration pattern (Meissner, 2008). It seems that the main drawback of using data from birds caught in walk-in traps is that trapping efficiency may differ not only between years, but also within a season due to many external factors (e.g. weather conditions, water level changes). Therefore, catching data should only be used with caution in studies of migration phenology in waders. On the other hand, it should be kept in mind that during the count, we do not know the extent to which we deal with individuals counted on a previous day when birds are counted every day.

The proportion of waders that are juveniles is often considered a good indicator of breeding success. Two methods can be used to obtain such data: counting or catching. However, catching methods may be particularly prone to bias, mainly toward juveniles due to their lower awareness and greater naivety. Moreover, depending on local conditions, the age structure of waders caught at least in walk-in traps may be biased towards juveniles or adults (Meissner, 2007). Therefore determining the proportion of juveniles from regular counts may be the better method of assessing wader productivity at stop-over sites during autumn migration than catching. However, the age structure monitored in any given season also partly depends on the length of birds' stopover at the study area, and the duration of stay may, and will, differ between adult and juvenile birds.

Wader counts should be conducted regularly in a standardized way and cover a large enough area so that bias arising from systematic differences in the distribution of adults and juveniles is minimised (Harrington, 2004). However, catching remains the main method for recording productivity in most species in winter (when the differences between adult and juvenile plumage become difficult to discern in the field) and in autumn (for species with only slight age-related differences in plumage).

To collect data on migration phenology or the changes of migrant numbers, every-day counts of waders resting and foraging in the surroundings of the station are recommended. If possible, at least two counts within every five-day period should track changes in bird numbers during migration. When waders are counted, adult and juvenile birds should be noted separately, if possible, in a given species. However, in stop-over sites with high turn-over rate, the number of caught birds may exceed the maximum number of seen during the day. In such cases, results of counts and catching should be analysed together. It is good practice to note the number of birds 
also during trap checking and other observations made during the day. It gives data on species, which appeared solitary or in very low number that might be not seen during the standard count. Only people that are capable of recognizing all species in the field and estimating the size of flocks should record counts. The best time for counting is around noon, after a few birds walk into the traps, and the majority of them are resting, which also makes counting easier. Remember to add the number of birds caught in the traps to the overall count! Many wader species have a tendency to prefer one particular type of habitat. For instance, e.g. curlew sandpipers or bartailed godwits are seldom met in a wet meadow, while, for example Sanderling and Turnstone practically always feed on sandy beaches. Therefore, it is preferred if the area where birds are counted includes information about all the habitats found in the immediate vicinity. A strict definition of the limits of the census area is important as well. The area should, if possible, have natural demarcations, and a meadow or a muddy bay should not be divided. On the other hand, the area must not be too large, because walking around it and counting the birds should not take more than 1-2 hours. If the count is done by a ringer, it should start immediately after all birds from the previous control walk have been ringed, so that birds brought from the next control walk will not have to wait for the ringer to return. A light telescope is very helpful in counts. It should be remembered, however, that some species (snipes) are very shy and spotting all individuals in the vegetation is practically impossible with a telescope. Therefore, places where snipes congregate should be walked over in a zigzag pattern, trying to flush all individuals on the wing.

Recently many colour-ringing schemes have been implemented for waders. It is worthwhile to pay attention to birds with such rings during the counts. 